Review

\title{
Heme oxygenase 1: a novel oncogene in multiple gynecological cancers
}

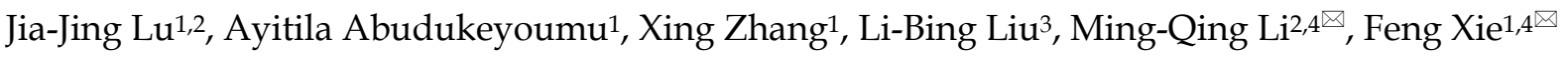 \\ 1. Medical Center of Diagnosis and Treatment for Cervical Diseases, Obstetrics and Gynecology Hospital of Fudan University, Shanghai 200011, People's \\ Republic of China. \\ 2. Laboratory for Reproductive Immunology, NHC Key Lab of Reproduction Regulation (Shanghai Institute of Planned Parenthood Research), Hospital of \\ Obstetrics and Gynecology, Fudan University, Shanghai 200080, People's Republic of China. \\ 3. Department of Gynecology, Changzhou No.2 People's Hospital, affiliated with Nanjing Medical University, Changzhou, Jiangsu Province, 213003, People's \\ Republic of China. \\ 4. Shanghai Key Laboratory of Female Reproductive Endocrine Related Diseases, Hospital of Obstetrics and Gynecology, Fudan University, Shanghai 200080, \\ People's Republic of China. \\ $\square$ Corresponding authors: Feng Xie, E-mail: fengxie10@fudan.edu.cn; or Ming-Qing Li, E-mail: mqli@fudan.edu.cn.
}

() The author(s). This is an open access article distributed under the terms of the Creative Commons Attribution License (https://creativecommons.org/licenses/by/4.0/). See http://ivyspring.com/terms for full terms and conditions.

Received: 2021.03.30; Accepted: 2021.05.24; Published: 2021.06.01

\begin{abstract}
Heme oxygenase 1 (HO-1), also known as heat shock protein 32 (HSP32), is a stress-inducible enzyme. In the past, it was believed to participate in maintaining cell homeostasis, reducing oxidative stress damage and exerting anti-apoptotic effects. When exposed to noxious stimulation, the expression of HO- 1 in the body will increase, antagonizing these oxidative stresses and protecting our bodies. Recently, many studies showed that HO-l was also highly-expressed in multiple gynecological cancers (such as ovarian cancer, cervical cancer and endometrial cancer), suggesting that it should be closely related to cell proliferation, metastasis, immune regulation and angiogenesis as an oncogene. This review summarizes the different effects of HO-l under normal and diseased conditions with a brief discussion of its implications on the diagnosis and treatment of gynecological cancers, aiming to provide a new clue for prevention and treatment of diseases.
\end{abstract}

Key words: Heme Oxygenase-1, proliferation, metastasis, cancer, oxidative stress

\section{Introduction}

The most important component of red blood cell is hemoglobin, which is composed of globin and heme [1]. Heme is a large complex, containing iron and protoporphyrin IX [2-4]. It is a cellular oxidant, participating in the formation of oxidative free radicals and leading to oxidative injury [5]. Noxious stimulation leads to the increase of heme oxygenase 1 (HO-1) for antagonizing these oxidative stresses and protecting our bodies [6]. Therefore, the hypothesis that HO-1 may be used as a targeted gene in tumor treatment has attracted more and more attentions. It is one of the most widely distributed antioxidative enzymes in the body and is the rate-limiting enzyme of heme metabolism [7].

Among all these malignant tumours, gynecological cancers is a specific type of fatal disease which only happens to women, and can seriously threaten the lives and health of women around the world. In recent years, many studies have confirmed that HO-1 is highly-expressed in a variety of gynecological tumors, such as ovarian cancer, cervical cancer and endometrial cancer. The elevated level of HO- 1 and the deviation of its dynamic trend from the baseline may be a signal of disease alert [8-11]. Studies have also shown that as a novel oncogene, HO-1 is closely related to tumor proliferation and metastasis, and may become a potential marker for predicting the prognosis of gynecological tumors [9]. Furthermore, HO-1 itself is also expected to become a target for tumor treatment. HO-1 inhibitors such as zinc protoporphyrin (ZnPP) have obtained certain efficacy in clinical work [12]. Therefore, this review summarizes the expression, regulation, roles and treatment values of HO-1 in gynecological tumors. 


\section{HO-1 and heme metabolism}

\section{HO family and HO-I}

In the long course of evolution, cells have developed a set of mechanisms against oxidative stress, and heme/HO system is one of the most important anti-oxidant mechanisms. Since Tenhunen and his colleagues first described the mechanism of heme catabolism in 1968 [13], people have gradually conducted more and more studies on the HO family. It is an unique type of cell protection enzyme located in the endoplasmic reticulum, and participate in the metabolism of heme [14].

From algae to humans, $\mathrm{HO}$ is ubiquitous and highly-conserved, suggesting that it may play an indispensable role in cells. We usually divide $\mathrm{HO}$ into three categories [15]. As shown in Table 1, HO-1 is a $32 \mathrm{kD}$ stress-inducible enzyme [16]. As it can be induced by high temperature, so it is also known as heat shock protein 32 (HSP-32) [13, 17]. Highexpression of HO-1 can be observed in liver, spleen, bone marrow and senescent erythrocytes $[18,19]$, and its main function is to degrade heme into biliverdin $(\mathrm{BV})$, carbon monoxide (CO) and ferrous ion $\left(\mathrm{Fe}^{2+}\right)$ $[20,21]$. Under the action of biliverdin reductive (BVR), BV will be further processed into bilirubin (BR) $[22,23]$. By regulating intracellular levels of heme and heme metabolites, HO-1 participates in maintaining cell homeostasis, reducing oxidative damage, regulating cell proliferation and apoptosis [24]. HO-2 is a $36 \mathrm{kD}$ constitutive enzyme, which is related to the nerve creed effect of carbon monoxide, it also participates in hemoglobin degradation [25]. High levels of HO-2 can be found in brain tissue, retina and testis [26]. Many scholars speculate that the presence of HO-2 in the testis may play a crucial role in the male reproductive system [27], but this hypothesis has not been confirmed. What has been verified is that HO-2 can protect neurons against ischemia/ reperfusion injury. HO-3 is widely distributed but has weak activity, which can promote the combination of heme and HO [28]. Sometimes it is viewed as a pseudogene processed from HO-2 transcription [26]. Among different $\mathrm{HO}$ isoforms, HO-1 seems to be the most valuable because its expression level can be induced under various pathophysiological conditions [24].

\section{Metabolites related to HO-1}

\section{Carbon monoxide}

As depicted in Figure 1, carbon monoxide (CO) is a gaseous product, most $\mathrm{CO}$ in our bodies comes from heme metabolism, it is an important signaling molecule [22, 29]. It can act directly on the blood vessels to cause vasodilation, this effect can also be achieved through the activity of the autonomic nervous system $[30,31]$. Besides, $C O$ plays a significant role in anti-apoptotic effects, this can be synergistic with other anti-apoptotic system [32, 33], by regulating the Mitogen-activated protein kinase (MAPK) signal pathway and inhibiting the activated mononuclear macrophage system. Exogenous $\mathrm{CO}$ has also this effect by up-regulating the expression of HO-1. Additionally, CO also participates is involved in many other physiological and pathological processes, for example, anti-inflammation, antiproliferation [16, 34], inhibition of platelet aggregation $[22,35]$ and neurotransmission [36].

\section{Biliverdin and bilirubin}

BVR is an NADPH-dependent enzyme, which can reduce biliverdin (BV) to bilirubin (BR) in the presence of NADPH. It is a dual-specific protein kinase that phosphorylates serine and tyrosine, therefore it's a key enzyme for protein phosphorylation. Apart from working as reductase and dual-specific protein kinase, BVR can also regulate tumor cells by acting as a basic leucine zipper (bZIP) transcription factor [37, 38].

The anti-apoptotic effect of BV and BR is achieved by promoting antiapoptotic protein bcl-2 and inhibiting pro-apoptotic protein Bax [39]. BR is also an important antioxidant in our bodies [40], it can inhibit the peroxidation of lipid and protein by scavenging excessive reactive oxygen species (ROS) [20, 41]. Oxidative stress is caused by the imbalance between cellular oxidants and antioxidants, ROS is a major oxidant in our bodies $[42,43]$, which can cause protein denaturation, genetic instability, and promote tumorigenesis [43]. It is usually generated by oxygen metabolism, but noxious stimulation can also cause an increase in ROS [44].

Table 1. Comparison of three $\mathrm{HO}$ isozymes

\begin{tabular}{lcc}
\hline & HO-1 & HO-2 \\
\hline $\begin{array}{l}\text { Molecular weight } \\
\text { Inducibility }\end{array}$ & $32 \mathrm{kD}$ & $36 \mathrm{kD}$ \\
Activity level & Stress-inducible & Constitutive \\
Highly-Expressed localization & High & High \\
Similarity & Live, spleen, bone marrow, senescent & Unknown \\
erythrocytes & Low
\end{tabular}




\section{Ferrous ion}

$\mathrm{Fe}^{2+}$ is cytotoxic because it can interact with cellular oxidants to generate ROS, promoting oxidation and inflammation. However, $\mathrm{Fe}^{2+}$ generated by heme metabolism can increase the expression of ferritin, which turns to prevent inflammation. In other words, when HO-1 is highly expressed, the level of $\mathrm{Fe}^{2+}$ increases, iron regulatory proteins (IRPs) can be dissociated from mRNA, which promotes ferritin translation. Ferritin increases the anti-injury ability of cells and makes the tumors more sensitive to oxidative stress, realizing the anti-tumor effect of HO-1 in iron metabolism. However, some studies pointed out that many diseases, such as Alzheimer, patients' lipid peroxidation may be related to iron accumulation. The amount of iron and ROS is the determinative momentum for the role of HO- 1 , excessive iron and ROS can turn HO-1 from a cell protector to a perpetrator, causing DNA damage, gene mutations and even cell death [45, 46]. Ferroptosis is a newly-identified non-programmed cell death, which is characterized by iron-overload and lipid peroxidation [47, 48]. Therefore, some researchers proposed that it can be used as a new strategy in many diseases, especially in cancer therapy.

\section{The role of HO-1 in different tumors}

Oxidative stress is caused by the imbalance between oxidation and antioxidant system in our bodies [49]. Common noxious stimuli include hypoxia, inflammatory cytokines, ultraviolet light, heavy metal ions, radiotherapy and so on [24, 47]. These stimuli changes the body's homeostasis, which triggers the activation of various signaling pathways, leading to disease progression. In normal cells, HO-1 acts as a cytoprotective agent, it can fight against oxidative injuries and regulate inflammatory response $[50,51]$. In ischemic diseases, HO-1 has been be used for the treatment of glucocorticoid-related osteoporosis and osteonecrosis [52]. Noxious stimuli actives upstream signal kinases, promoting the binding of DNA and transcription factors, which results in high expression of $\mathrm{HO}-1$ and providing protection for normal cells [50,53].

Nevertheless, the biological effect of HO-1 seems to be tissue-specific. In some cancer cells, HO-1 plays the role of survival factor [29]. Overexpression of HO-1 promotes tumor progression in turn [23, 54]. It can facilitate angiogenesis and prevent tumor cells from apoptosis, leading to its survival and progression [55]. Researches suggest that heme can induce a stress-inducible protein Sestrin2 (SESN2), which is a protective mechanism to antagonize oxidative stress and colon tumor growth. However, high level of SESN2 can promote tumorigenesis [56]. Another high-risk factor of colorectal carcer is the consumption of red meat. Heme iron is the main component of red meat, which may be the cause of excessive colonic proliferation and carcinogenesis [57]. Antioxidants can stimulate lung cancer metastasis by reducing heme levels and stabilizing the transcription factor $\mathrm{BTB}$ and $\mathrm{CNC}$ homology 1 (BACH1) [58]. Overexpression of HO-1 induces the expression of Cyclin Dependent Kinase 4 (CDK4) and promotes the occurrence of liver cancer; however, a feedback loop may exist between IL-6 and HO-1, thus HO-1 can be induced as an antitumor gene through the IL-6/JAK/STAT3 pathways [59, 60]. The application of HO-1 inhibitor zinc protoporphyrin (ZnPPIX) can greatly inhibit the proliferation of pancreatic cancer cells, while HO-1 significantly promotes cell proliferation [61]. Overexpression HO-1 promotes the occurrence of melanoma and plays the role of anti-apoptosis through the B-Raf-ERK signaling pathway [62]. The most common metastatic site of prostate cancer is bone, a research by Anselmino has showed that HO-1 is a pivotal modulator of bone turnover and remodeling because it can promote the growth and invasion of cancer cells both in vivo and in vitro. This may be related to the epithelial-mesenchymal transition (EMT) induction and antioxidant and antiapoptotic effects of the prostate cancer cells $[63,64]$.
Figure 1. Heme metabolism and its main metabolites. Oxidative stress increases the level of $\mathrm{HO}-1$, which can degrade heme into $\mathrm{BV}, \mathrm{BR}, \mathrm{CO}$ and $\mathrm{Fe}^{2+}$. Through regulating the autonomic nervous system, $\mathrm{CO}$ can act directly on the blood vessels to cause vasodilation. Large amount of $\mathrm{Fe}^{2+}$ generated by heme metabolism can dissociate IRPs from mRNA and promote the translation of ferritin, which increases the anti-injury ability of cells and makes the tumors more sensitive to therapy. BVR is an NADPH-dependent enzyme, and it can reduce BV into BR. BV and BR then work together to scavenge excessive ROS and inhibit the peroxidation of lipid and protein. In summary, by regulating intracellular levels of heme and heme metabolites, $\mathrm{HO}-1$ participates in maintaining cell homeostasis, reducing oxidative damage, regulating cell proliferation and apoptosis. HO-1: Heme oxygenase 1; BV: biliverdin; BR: bilirubin; CO: carbon monoxide; $\mathrm{Fe}^{2+}$ : ferrous ion; IRPs: iron regulatory proteins; BVR: biliverdin reductive; ROS: reactive oxygen species. 
HO-1 can increase anti-apoptotic ability, which may lead to their uncontrolled cell proliferation and even cause tumorigenesis [24]. Compared with the surrounding normal tissues, therefore, the increased expression of HO-1 can be observed in tumors [11]. Abnormal signaling pathway activation can lead to reduced self-adhesion ability of tumor cells, tumor angiogenesis and changes in microenvironment [65], preventing the cancer cells from apoptosis and autophagy, and even promoting their proliferation and metastasis [47]. Many studies have shown that HO-1 is a crucial substance for angiogenesis [66], which can help malignant tumors continue to grow and invasion. Currently, highly-expressed HO-1 has been found in various malignant tumors such as melanoma [2], thyroid cancer [5], osteosarcoma [50], breast cancer [67], lung cancer [58], bowel cancer [56, 57, 68], renal cell cancer [69], hepatoma [59], prostate cancers [63, 64], pancreatic cancer [61], and so on.

However, the formation of tumors is not only related to the cancer cells themselves. In fact, their occurrence, growth and even metastasis are very closely related to the surrounding cells (immune/ inflammatory cells, glial cells, fibroblasts, etc.) and extracellular components (cytokines, growth factors, hormones, etc.), namely the so-called tumor microenvironment (TME) [70, 71]. More and more studies have shown that HO-1 can affect cancer progression through modulating TME [72]. Of note, HO-1 can act as an immunomodulator that inhibits cell maturation, activation and infiltration [73-75]. Myeloid-derived suppressor cells (MDSCs) are known to inhibit anti-tumor immunity, HO-1 expression in MDSCs plays a role in the suppression of alloreactive $T$ cells $[76,77]$ through promoting the release of many inflammatory factors such as interleukin-10 (IL-10) [78] and tumor necrosis factor- $\alpha$ (TNF- $\alpha$ ) [79], the expression of transforming growth factor $\beta$ (TGF- $\beta$ ), intercellular adhesion molecule 1 , and other fibrogenesis factors increase, activating the NF-kB/Signal Transducer and Activator of Transcription (STAT) 3 signaling pathway [80, 81], and maintaining the self-renewal ability of cancer stem cells[82]. By regulating the inflammatory response and anti-tumor immunity, these immune/ inflammatory cells play significant roles in TME, which can deeply affect cancer progression.

Meanwhile, as a key mediator of angiogenesis, vascular endothelial growth factor (VEGF) can form new vasculature around tumors, causing them to grow exponentially [83]. HO-1 also participates in fostering angiogenesis linked to inflammation and tumor by up-regulating the expression of VEGF in macrophages. A study conducted by Gabriel et al. has showed that the ectopic expression of HO-1 can significantly increase the transcriptional activity of VEGF in prostate cancer cells [84]. In addition, VEGF fosters the formation of capillary-like tubular structures in tumor tissues $[85,86]$, and promotes the proliferation and migration of cancer cells.

More importantly, the level of HO-1 is closely related to the clinical features and prognosis of tumors. Generally speaking, the higher the expression of HO-1, the lower the tumor differentiation, and the more active the proliferation and metastasis [9]. HO-1 inhibitors can suppress cell proliferation and invasion by increasing intracellular ROS levels and inducing cell cycle arrest [5]. Because HO-1 is so closely related to tumors, some people even proposed that $\mathrm{HO}-1$ can also be used as one of the tumor markers. However, a study on breast cancer showed that HO-1 overexpression can reduce lung metastasis by inhibiting cell EMT and proliferation, suggesting that HO-1 is tissue-specific [87], which needs to be studied further.

HO-1 plays different roles during different stages of tumor formation. Before a tumor is formed, it can remove aging and dead cells, inhibit tumors and protect normal cells. When a tumor is formed, the activation of HO-1 enables tumor cells to gain this anti-apoptotic ability, which leads to the occurrence and proliferation of tumors [53]. In that case, it has a protective effect on tumor cells instead.

\section{Regulation mechanism of HO-1 expression}

Different HO-1 inducers activate different protein phosphorylation-dependent signaling pathways, then activate various transcription factors. MAPK is one of the most important signal kinases in HO-1 transcription, other signal kinases such as phosphatidylinositol 3-kinase (PI3K), tyrosine kinases and many protein kinases (PK) also participate in this process [24]. The latest study suggests that the regulation of its enzymatic activity depends heavily upon the expression of transcriptional level [88].

Nuclear factor E2-related factor 2 (Nrf2) is a key transcription factor involving in maintaining cell redox homeostasis [89], and HO-1 is one of the most important regulatory products. It is a bZIP transcription factor [10]. Under resting conditions, Nrf2 binds to kelch-like ECH-related protein 1 (keap1) and form a Keap1-Nrf2 complex [90]. The complex will be degraded by ubiquitous proteasome and exist in the cytoplasm in an inactive state. However, oxidative stress induces the modification of cysteine residues in Keap1, causing Nrf2 to dissociate from the complex and increase the translocation of nucleus [10]. Within the nucleus, it binds to the antioxidantresponsive element (ARE) in target gene promoters 
and form the Nrf2-ARE signaling pathway [90], activating the transcription of its downstream target genes, such as HO-1 and NADPH quinone dehydrogenase 1 (NQO1), protecting cells from oxidative damage and participating in maintaining redox homeostasis $[24,42,56]$.

The PI3K/protein kinase B (PKB, also known as AKT) signaling is one of the most critical pathways in regulating cell growth, proliferation and apoptosis. Studies have found that Nrf2 is significantly elevated in tumor cells. Inactivating PI3K/AKT pathway can significantly reduce the level of Nrf2, inhibiting tumor cell proliferation, inducing cell apoptosis, and improving the sensitivity of tumor cells to treatment [91]. In addition, MAPK signaling pathways can also regulate the activity of Nrf2.

Nuclear factor $\mathrm{\kappa B}(\mathrm{NF}-\mathrm{kB})$ and Bach1 also play a key role in the occurrence and development of cancer [92], and is considered to be a target for the cancer therapy. Under resting conditions, IкB binds to NF-кB and Bach1 binds to Maf recognition element (MARE), they form a new complex respectively, preventing NF-kB and Bach1 translocation from the cytoplasm to the nucleus [93]. Once oxidative stress stimulates the complex, NF- $\kappa B$ dissociates from IkB, Bach1 dissociates from MARE. Both of them can activate the transcription of HO-1 [18, 43].

\section{HO-1 and gynecological tumors}

\section{Ovarian caner}

In early stage of ovarian cancer (OC), patients lack specific manifestations, so early lesion is very easy to neglect. When patients show symptoms related to OC, they're usually at their advanced stage, tumor progresses rapidly, plus no effective treatment, therefore, the fatality rate of OC ranks first among gynecological malignancies [48].

Just as HO- 1 is elevated in many tumors, the level of HO-1 in ovarian cancer is higher than that in normal ovarian tissues, which may be achieved by activating the VEGF. The VEGF/VEGFR signaling pathway is a key regulator of tumor angiogenesis, upregulating VEGFR2 can significantly increase the level of HO-1, which is a downstream target gene of Nrf2 $[8,94]$.

Apatinib is a novel tyrosine kinase inhibitor, which can specifically target vascular endothelial growth factor receptor 2 (VEGFR2) and maintain it at a low level in OC cells, inhibiting the migration and proliferation of endothelial cells induced by VEGFR2 [66]. A study found that after Apatinib treatment, the levels of pro-apoptotic protein bax in OC cells increased, while the expression of p62 and anti-apoptotic protein $\mathrm{Bcl}-2$ reduced [95]. Light chain 3 (LC3) is an autophagy marker. When the cell undergoes autophagy, the cytoplasmic type (LC3-I) will decompose a small piece of peptide and transform into the membrane type (LC3-II). Therefore, the ratio of LC3-II/I can be used to evaluate the level of cell autophagy. In this experiment, the conversion of LC3-I to LC3-II also increased [9]. This may be because Apatinib can act directly on OC cells, reducing tumor microvessel density, down-regulating the Nrf2/HO-1 pathway [93, 96], and promoting glutathione to generate ROS [97]. In short, inhibiting Nrf-2/HO-1 pathway promotes ROS-dependent apoptosis and autophagy in OC cells [66, 93, 95], and plays an anti-tumor role [10].

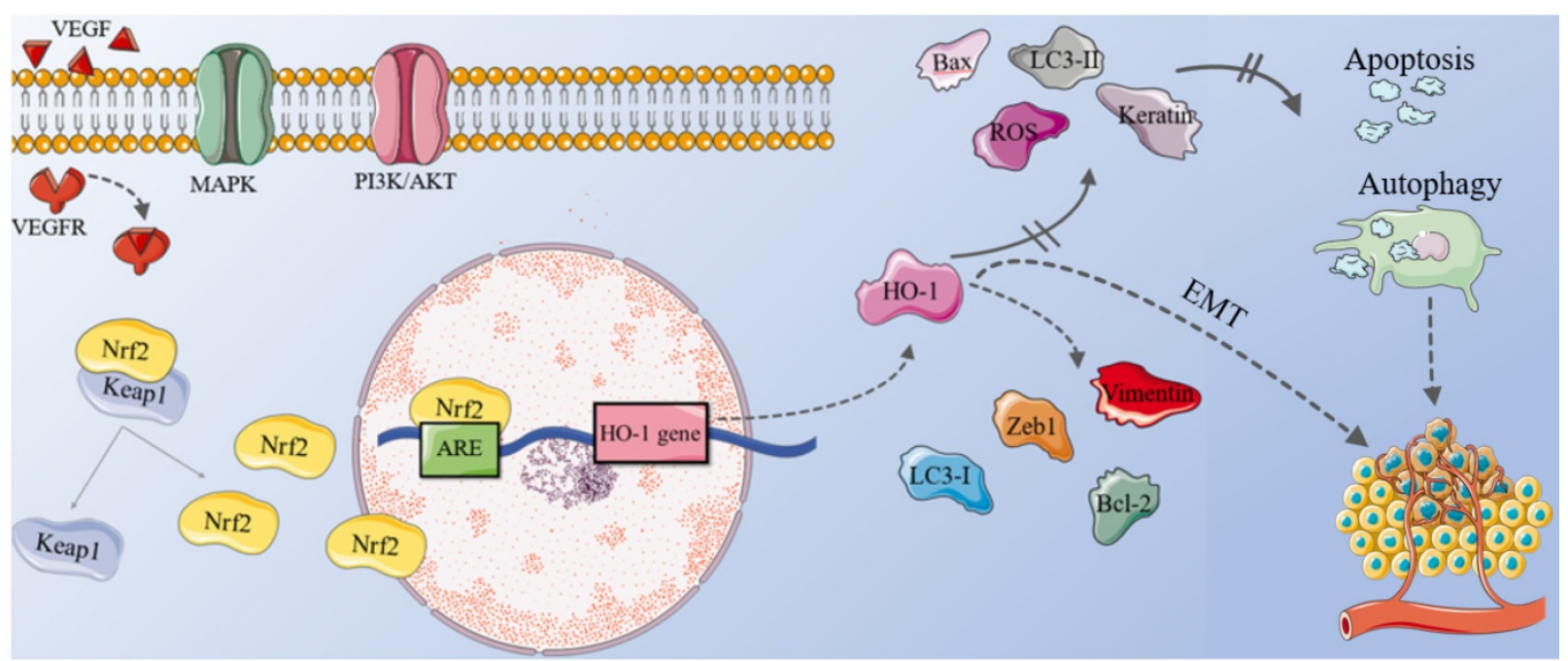

Figure 2. The mechanism of HO-1 in ovarian cancer. In OC cells, the combination of VEGF and VEGFR, plus the abnormal activation of MAPK and PI3K/ AKT signaling pathways can cause Nrf2 to dissociate from the Nrf2-Keapl complex and increase the translocation of nucleus. Within the nucleus, Nrf2 binds to ARE, activating the transcription of its downstream gene HO-1. High levels of LC3-I, vimentin, Zebl and Bcl-2 can be observed in OC cells, while the level of LC3-II, keratin, Bax decrease. Therefore, up-regulating of the Nrf2/HO-1 pathway inhibits ROS-dependent apoptosis and autophagy in OC cells, increasing tumor microvessel density and promoting the growth and metastasis of OC. EMT also participate in this process collaboratively. OC: ovarian cancer; VEGF: vascular endothelial growth factor; VEGFR: VEGF receptor; Nrf2: nuclear factor E2-related factor 2; Keap1: kelch-like ECH-related protein 1; ARE: antioxidant-responsive element; LC3: light chain3; EMT: epithelial-mesenchymal transition. 
EMT plays a key role in the growth and metastasis of tumor. HO-1 promotes the proliferation and migration of ovarian cancer cells by affecting EMT [98]. Therefore, the level of HO-1 is closely related to its lymph node metastasis and FIGO stage. After treating OC tissue with heme inducer for 24 hours, vimentin (a mesenchymal marker), Zeb1 (a EMT transcription factor) and anti-apoptotic protein Bcl-2 were up-regulated; the levels of keratin and pro-apoptotic protein Bax decreased. The result is opposite after treating OC tissue with $\mathrm{HO}-1$ inhibitor Znpp for 24 hours [9], suggesting that patients with high HO-1 expression have poorer prognosis and much lower overall survival.

In addition, endometriosis can lead to endometriosis-associated ovarian cancer (EAOC) [99], although this malignant transformation is relatively rare [48]. Studies have shown that the number of M2 macrophages expressing $\mathrm{HO}-1$ in EAOC is significantly reduced [100], suggesting that redox imbalance may participate in the malignant transformation of endometriosis [54]. However, more research is still needed to confirm this theory.

\section{Cervical cancer}

Cervical cancer (CC) is the most common female malignancy with a high incidence among $50-55$ yearsold women. However, in recent years, patients tend to be younger and younger [91]. When squamous intraepithelial lesion (SIL) forms and continues to develop, it can exceed the basal layer, infiltrate the interstitial tissues and form invasive carcinoma.

Human papilloma virus (HPV) infection is the most important cause of CC. The oncoprotein produced by HPV may lead to autophagic dysfunction and the hinder viral clearance. Studies have found that $\mathrm{HO}-1$ is overexpressed in HPV-infected tissues, especially in low-grade cervical intraepithelial neoplasia (CIN) lesions. X-linked inhibitor of apoptosis (XIAP) is an anti-apoptotic molecule and is considered to be a negative regulator of autophagy. By downregulating the expression of XIAP, HO-1 also increases the ratio of LC3-II/I [101].

On the other hand, HO-1 is a target gene of Nrf2, and its elevated level leads to the overexpression of Nrf2 in CC cells, which promotes the proliferation and invasion ability of SiHa cells [42], and makes tumor cells become insensitive to treatment [42, 91]. Meanwhile, high levels of HO-1 are closely related to the clinical staging of cervical cancer, lymph node metastasis and poor prognosis.

Knockout of HO-1 can stimulate the inherent cellular response, promote autophagy response of CC cells, and downregulate the expression of antiapoptotic proteins through anti-viral mechanisms.
Hyperthermia is one of the adjuvant therapies for cervical cancer [17]. A research showed that hyperthermia could down-regulate the expression of HO-1 in cervical cancer cells, reduce the viral load of HPV16 E6, and even destroy the existing physical state of HPV16. The combination of the two methods has a better effect than a single one [101].

\section{Endometrial cancer}

Endometrial cancer (EC) usually occurs in elderly women, irregular vaginal bleeding after menopause is a typical symptom. EC can be divided into 2 types, type 1 refers to endometrioid carcinoma, which accounts for $75 \%$. As an estrogen-dependent type, it's closely associated with hyperplastic proliferation of the endometrial glands [102]. Compared with normal tissues, endometrial hyperplasia $(\mathrm{EH})$ is characterized by an increased ratio of endometrial glands to stroma by more than 1:1.Dysplasia of endometrium is even considered to be precancerous lesions, up to $50 \%$ patients will finally develop into EC [103, 104]. A study carried by Fatma et al. has showed that increasing the expression of HO-1 can ameliorate the $\mathrm{EH}$ induced by estrogen, which maybe achieved by the suppression of inducible nitric oxide synthase (iNOS), p38, MAPK, and Ki67 [43]. However, in EC tissues, HO-1 is highly expressed. This is because EC often has irregular vaginal bleeding, which may be related to the massive release of heme. As is mentioned before, the degration porducts of heme are potentially toxic. Through mediating oxidative stress and inflammatory stimuli, large amount of heme in turn accelerates the occurrence and development of tumors [53]. Overall, HO- 1 shows completely different effects in EH tissues and in EC. However, articles on the role of HO-1 in EC are very limited and its mechanism is still unclear, which is worthy of further investigation.

\section{HO-1 and treatment insensitivity}

Chemotherapy is a very important adjuvant treatment method after malignant tumor surgeries [89]. Many commonly-used chemotherapy drugs can generate ROS to achieve the purpose of inducing cancer cell apoptosis $[65,96,98]$. In normal situations, antioxidants can protect our body from oxidative stress damage; nevertheless, cancer cells may also activate antioxidant signals to fight against the damage caused by chemotherapy drugs, namely, cells develop drug resistance [105]. Cis-platinum (CDDP) is one of the most widely used chemotherapy drugs [8]. Recent reports indicated that its cytotoxic effect may be achieved by ROS-dependent apoptosis or DNA damage $[17,106]$. Other people believe that this may because chemotherapy-generated tumor cell 
debris hijack tumor-associated macrophages (TAMs). By promoting HO-1 expression and reducing M1-like polarization, tumor cells developed resistance to chemotherapy drugs. What's more, overexpression of HO-1 is often accompanied by an increase in multidrug resistance-related proteins (Mrp), which is an important reason for the difficulty in tumor treatment [67].

HO-1 plays a protective role in tumor cells has been widely recognized [107]. Studies have found that HO-1 inhibitor ZnPPIX can improve sensitivity to chemotherapy of gastric cancer cell [12, 108, 109], other research showed that the inhibition rate of esophageal cancer cells was positively correlated with ZnPPIX concentration [109, 110]. A study based on the treatment of acute myelogenous leukemia (AML) has found that through non-covalently modified, lipidpolymer hybrid nanoparticle loaded with HO1inhibitor tin mesoporphyrin (SnMP) can significantly improve the efficacy of daunorubicin and boost immune response [17, 111]. Furthermore, a strong potential of blocking HO-1 for the treatment of hereditary leiomyomatosis and renal cell carcinoma (HLRCC) has already been verified [69]. All these findings remind us that exploring the possibility of targeting or genetically or pharmacologically inhibiting HO-1 could make immunotherapy more effective [112], and HO-1 inhibitor may be used as a potential chemotherapeutic sensitizer in the near future [113].

\section{Conclusion and Prospective}

Heme/HO system is one of the most important anti-oxidant mechanisms in our bodies. As a potential novel oncogene, HO-1 has received increasing levels of attention in recent years. On the one hand, HO- 1 is highly expressed in a variety of gynecological malignancies, so the deviation of its dynamic trend from baseline could be used as a signal of disease alert and a predictor for the occurrence of tumors. On the other hand, for oncology patients with clinical manifestations and imaging evidence, high-level of HO-1 can also assist diagnosis. HO-1 level has certain relevance with prognosis and can be used as a potential indicator. Through inhibiting HO-1 directly or indirectly, HO-1 inhibitors can promote ROS-dependent autophagy and apoptosis. At present, HO-1 inhibitors have been used in clinical work and achieved certain efficacy.

However, the exact mechanism of HO-1 in gynecological tumors is still unclear. For one thing, HO-1 was proposed as a novel oncogene in gynecological malignancies not long ago, thus it has not yet been fully studied. For another, HO-1 seems to be tissue-specific. In normal tissues, it plays the role of anti-inflammation and anti-apoptosis, which indicates us to seek for non-stress HO- 1 inducers for body protection. However, in cancer cells, HO-1 facilitates angiogenesis and tumor metastasis in turn. The contradiction makes the research on HO-1 very difficultly.

Anyway, regulating the expression of $\mathrm{HO}-1$ may be a potential target of clinical treatment for patients with gynecological malignancies, although further studies are still needed to be done. Clarifing the exact mechanism of HO-1 in gyncological cancers may pave a new way for preventing the onset or progression of gyncological cancers.

\section{Acknowledgements}

\section{Funding}

This study supported by the National Natural Science Foundation of China (NSFC) (No. 82072872), the Innovation-oriented Science and Technology Grant from NPFPC Key Laboratory of Reproduction Regulation (CX2017-2), the Program for Zhuoxue of Fudan University (JIF157602), the Support Project for Original Personalized Research of Fudan University, the Open Project Program of Shanghai Key Laboratory of Female Reproductive EndocrineRelated Diseases (grant no. 17DZ2273600) and the Affiliated Changzhou No. 2 People's Hospital of Nanjing Medical University (2018K006).

\section{Authors' contributions}

J.J.L. performed the literature research, wrote the manuscript and prepared the table and figures. A.A., X.Z., and L.B.L. helped to perform revisions and critically discussed the complete manuscript. F.X. and M.Q.L. designed and wrote the manuscript, supervised, and critically reviewed the complete manuscript. All authors have approved it for publication.

\section{Competing Interests}

The authors have declared that no competing interest exists.

\section{References}

1. Andersen CBF, K Stødkilde, KL Sæderup, A Kuhlee, S Raunser, et al. Haptoglobin [J]. Antioxid Redox Signal. 2017; 26: 814-31.

2. Jasmer KJ, J Hou, P Mannino, JL Cheng, M Hannink. Heme oxygenase promotes B-Raf-dependent melanosphere formation [J]. Pigment Cell \& Melanoma Research. 2020; 33: 850-68.

3. Canesin G, SM Hejazi, KD Swanson, B Wegiel. Heme-Derived Metabolic Signals Dictate Immune Responses [J]. Front Immunol. 2020; 11: 66-70.

4. Bonkovsky HL, JT Guo, W Hou, T Li, T Narang, et al. Porphyrin and heme metabolism and the porphyrias [J]. Compr Physiol. 2013; 3: 365-401.

5. Yang PS, YC Hsu, JJ Lee, MJ Chen, SY Huang, et al. Heme Oxygenase-1 Inhibitors Induce Cell Cycle Arrest and Suppress Tumor Growth in Thyroid Cancer Cells [J]. International Journal of Molecular Sciences. 2018; 19:134-38 
6. Drummond GS, J Baum, M Greenberg, D Lewis, NG Abraham. HO-1 overexpression and underexpression: Clinical implications [J]. Arch Biochem Biophys. 2019; 673: 108073.

7. Ohgari Y, Y Miyata, T Miyagi, S Gotoh, T Ohta, et al. Roles of porphyrin and iron metabolisms in the $\delta$-aminolevulinic acid (ALA)-induced accumulation of protoporphyrin and photodamage of tumor cells [J]. Photochem Photobiol. 2011; 87: 1138-45.

8. Bao LJ, MC Jaramillo, ZB Zhang, YX Zheng, M Yao, et al. Nrf2 induces cisplatin resistance through activation of autophagy in ovarian carcinoma [J]. Int J Clin Exp Pathol. 2014; 7: 1502-13.

9. Zhao Z, Y Xu, J Lu, J Xue, P Liu. High expression of HO-1 predicts poor prognosis of ovarian cancer patients and promotes proliferation and aggressiveness of ovarian cancer cells [J]. Clin Transl Oncol. 2018; 20: 491-99.

10. Xia MH, XY Yan, L Zhou, L Xu, LC Zhang, et al. p62 Suppressed VK3-induced Oxidative Damage Through Keap1/Nrf2 Pathway In Human Ovarian Cancer Cells [J]. J Cancer. 2020; 11: 1299-307.

11. Gómez-Lomelí P, A Bravo-Cuellar, G Hernández-Flores, LF Jave-Suárez, A Aguilar-Lemarroy, et al. Increase of IFN- $\gamma$ and TNF-a production in CD107a + NK-92 cells co-cultured with cervical cancer cell lines pre-treated with the HO-1 inhibitor [J]. Cancer Cell Int. 2014; 14: 100-14.

12. Fang J, K Tsukigawa, L Liao, H Yin, K Eguchi, et al. Styrene-maleic acid-copolymer conjugated zinc protoporphyrin as a candidate drug for tumor-targeted therapy and imaging [J]. J Drug Target. 2016; 24: 399-407.

13. Morse D, AM Choi. Heme oxygenase-1: from bench to bedside [J]. Am J Respir Crit Care Med. 2005; 172: 660-70.

14. Fernández-Fierro A, SC Funes, M Rios, C Covián, J González, et al. Immune Modulation by Inhibitors of the HO System [J]. Int J Mol Sci. 2020; 22:345-49

15. Pittalà V, L Salerno, G Romeo, MN Modica, MA Siracusa. A focus on heme oxygenase-1 (HO-1) inhibitors [J]. Curr Med Chem. 2013; 20: 3711-32.

16. Knauert M, S Vangala, M Haslip, PJ Lee. Therapeutic applications of carbon monoxide [J]. Oxid Med Cell Longev. 2013; 2013: 360815.

17. Cesna V, A Sukovas, A Jasukaitiene, G Silkuniene, S Paskauskas, et al. Stimulated upregulation of $\mathrm{HO}-1$ is associated with inadequate response of gastric and ovarian cancer cell lines to hyperthermia and cisplatin treatment [J]. Oncol Lett. 2019; 18: 1961-68.

18. Sudan K, V Vijayan, K Madyaningrana, F Gueler, K Igarashi, et al. TLR4 activation alters labile heme levels to regulate $\mathrm{BACH} 1$ and heme oxygenase-1 expression in macrophages [J]. Free Radic Biol Med. 2019; 137: $131-42$

19. Chen J. Heme oxygenase in neuroprotection: from mechanisms to therapeutic implications [J]. Rev Neurosci. 2014; 25: 269-80.

20. Maruhashi T, Y Kihara, Y Higashi. Bilirubin and Endothelial Function [J]. J Atheroscler Thromb. 2019; 26: 688-96.

21. Li Volti G, D Tibullo, L Vanella, C Giallongo, F Di Raimondo, et al. The Heme Oxygenase System in Hematological Malignancies [J]. Antioxid Redox Signal. 2017; 27: 363-77.

22. Ryter SW. Heme oxygenase-1/carbon monoxide as modulators of autophagy and inflammation [J]. Arch Biochem Biophys. 2019; 678: 108186-95.

23. Salerno L, G Floresta, V Ciaffaglione, D Gentile, F Margani, et al. Progress in the development of selective heme oxygenase- 1 inhibitors and their potential therapeutic application [J]. Eur J Med Chem. 2019; 167: 439-53.

24. Waza AA, Z Hamid, S Ali, SA Bhat, MA Bhat. A review on heme oxygenase-1 induction: is it a necessary evil [J]. Inflamm Res. 2018; 67: 579-88.

25. Liu L, AB Dumbrepatil, AS Fleischhacker, ENG Marsh, SW Ragsdale. Heme oxygenase-2 is post-translationally regulated by heme occupancy in the catalytic site [J]. J Biol Chem. 2020; 295: 17227-40.

26. Intagliata S, L Salerno, V Ciaffaglione, C Leonardi, AN Fallica, et al. Heme Oxygenase-2 (HO-2) as a therapeutic target: Activators and inhibitors [J]. Eur J Med Chem. 2019; 183: 111703-27.

27. Muñoz-Sánchez J, ME Chánez-Cárdenas. A review on hemeoxygenase-2: focus on cellular protection and oxygen response [J]. Oxid Med Cell Longev. 2014; 2014: 604981-89.

28. Hayashi S, Y Omata, H Sakamoto, Y Higashimoto, $\mathrm{T}$ Hara, et al. Characterization of rat heme oxygenase- 3 gene. Implication of processed pseudogenes derived from heme oxygenase-2 gene [J]. Gene. 2004; 336: 241-50.

29. Gáll T, G Balla, J Balla. Heme, Heme Oxygenase, and Endoplasmic Reticulum Stress-A New Insight into the Pathophysiology of Vascular Diseases [J]. Int J Mol Sci. 2019; 20:245-53

30. Castracani CC, L Longhitano, A Distefano, MD Rosa, V Pittalà, et al. Heme Oxygenase-1 and Carbon Monoxide Regulate Growth and Progression in Glioblastoma Cells [J]. Molecular Neurobiology. 2020; 57:228-35
31. Jung E, SH Koh, M Yoo, YK Choi. Regenerative Potential of Carbon Monoxide in Adult Neural Circuits of the Central Nervous System [J]. Int J Mol Sci. 2020; 21:342-46

32. Motterlini R, LE Otterbein. The therapeutic potential of carbon monoxide [J]. Nat Rev Drug Discov. 2010; 9: 728-43.

33. Takeda TA, M Sasai, Y Adachi, K Ohnishi, JI Fujisawa, et al. Potential role of heme metabolism in the inducible expression of heme oxygenase-1 [J]. Biochim Biophys Acta Gen Subj. 2017; 1861: 1813-24.

34. Cheng Y, J Rong. Therapeutic Potential of Heme Oxygenase-1/carbon Monoxide System Against Ischemia-Reperfusion Injury [J]. Curr Pharm Des. 2017; 23: 3884-98.

35. Nielsen VG, E Pretorius. Carbon monoxide: Anticoagulant or procoagulant? [J]. Thromb Res. 2014; 133: 315-21.

36. Fagone $\mathrm{P}, \mathrm{K}$ Mangano, M Coco, V Perciavalle, G Garotta, et al. Therapeutic potential of carbon monoxide in multiple sclerosis [J]. Clin Exp Immunol. 2012; 167: 179-87.

37. Mhillaj E, A Tarozzi, L Pruccoli, V Cuomo, L Trabace, et al. Curcumin and Heme Oxygenase: Neuroprotection and Beyond [J]. Int J Mol Sci. 2019; 20:357-68

38. Fujiwara R, M Haag, E Schaeffeler, AT Nies, UM Zanger, et al. Systemic regulation of bilirubin homeostasis: Potential benefits of hyperbilirubinemia [J]. Hepatology. 2018; 67: 1609-19.

39. Vítek L, C Tiribelli. Bilirubin, Intestinal Integrity, the Microbiome, and Inflammation [J]. N Engl J Med. 2020; 383: 684-86.

40. Vasavda C, R Kothari, AP Malla, R Tokhunts, A Lin, et al. Bilirubin Links Heme Metabolism to Neuroprotection by Scavenging Superoxide [J]. Cell Chem Biol. 2019; 26: 1450-60.e7.

41. Baylor JL, MW Butler. Immune challenge-induced oxidative damage may be mitigated by biliverdin [J]. J Exp Biol. 2019; 222:786-96

42. Zhang Q, D Yang. Allicin suppresses the migration and invasion in cervical cancer cells mainly by inhibiting NRF2 [J]. Exp Ther Med. 2019; 17: 1523-28.

43. Ali FF, WY Abdelzaher, RA Ibrahim, DM Elroby Ali. Amelioration of estrogen-induced endometrial hyperplasia in female rats by hemin via heme-oxygenase-1 expression, suppression of iNOS, p38 MAPK, and Ki67 [J]. Can J Physiol Pharmacol. 2019; 97: 1159-68.

44. Tabata S, R Ikeda, M Yamamoto, T Furukawa, T Kuramoto, et al. Thymidine phosphorylase enhances reactive oxygen species generation and interleukin-8 expression in human cancer cells [J]. Oncol Rep. 2012; 28: 895-902

45. Kwon MY, E Park, SJ Lee, SW Chung. Heme oxygenase-1 accelerates erastin-induced ferroptotic cell death [J]. Oncotarget. 2015; 6: 24393-403.

46. Kajarabille N, GO Latunde-Dada. Programmed Cell-Death by Ferroptosis: Antioxidants as Mitigators [J]. Int J Mol Sci. 2019; 20:882-97.

47. Chiang SK, SE Chen, LC Chang. A Dual Role of Heme Oxygenase-1 in Cancer Cells [J]. Int J Mol Sci. 2018; 20:568-76

48. Bauckman KA, E Haller, I Flores, M Nanjundan. Iron modulates cell survival in a Ras- and MAPK-dependent manner in ovarian cells [J]. Cell Death Dis. 2013; 4: 592-98.

49. Kletzmayr A, F Clement Frey, M Zimmermann, D Eberli, C Millan. An Automatable Hydrogel Culture Platform for Evaluating Efficacy of Antibody-Based Therapeutics in Overcoming Chemoresistance [J]. Biotechnol J. 2020; 15: 190-97.

50. Kharb S, M Halder, ZS Kundu. Heme oxygenase-1 in osteosarcoma [J]. J Cancer Res Ther. 2020; 16: 874-77.

51. Lever JM, R Boddu, JF George, A Agarwal. Heme Oxygenase- 1 in Kidney Health and Disease [J]. Antioxid Redox Signal. 2016; 25: 165-83.

52. Yamamoto H, M Saito, T Goto, K Ueshima, M Ishida, et al. Heme oxygenase-1 prevents glucocorticoid and hypoxia-induced apoptosis and necrosis of osteocyte-like cells [J]. Med Mol Morphol. 2019; 52: 173-80.

53. Wagener FA, HD Volk, D Willis, NG Abraham, MP Soares, et al. Different faces of the heme-heme oxygenase system in inflammation [J]. Pharmacol Rev. 2003; 55: 551-71.

54. Fujimoto Y, S Imanaka, Y Yamada, K Ogawa, F Ito, et al. Comparison of redox parameters in ovarian endometrioma and its malignant transformation [J]. Oncol Lett. 2018; 16: 5257-64.

55. Sferrazzo G, MD Rosa, E Barone, GL Volti, N Musso, et al. Heme Oxygenase-1 in Central Nervous System Malignancies [J]. Journal of Clinical Medicine. 2020; 9:783-95.

56. Kim H, K Yin, DM Falcon, $X$ Xue. The interaction of Hemin and Sestrin2 modulates oxidative stress and colon tumor growth [J]. Toxicol Appl Pharmacol. 2019; 374: 77-85.

57. Seiwert N, D Heylmann, S Hasselwander, J Fahrer. Mechanism of colorectal carcinogenesis triggered by heme iron from red meat [J]. Biochim Biophys Acta Rev Cancer. 2020; 1873: 188334-46.

58. Wiel C, K Le Gal, MX Ibrahim, CA Jahangir, M Kashif, et al. BACH1 Stabilization by Antioxidants Stimulates Lung Cancer Metastasis [J]. Cell. 2019; 178: 330-45. 
59. Chiang K-C, K-S Chang, S-Y Hsu, H-C Sung, T-H Feng, et al. Human Heme Oxygenase-1 Induced by Interleukin-6 via JAK/STAT3 Pathways Is a Tumor Suppressor Gene in Hepatoma Cells [J]. Antioxidants. 2020; 9:569-78.

60. Xi J, Y Sun, M Zhang, Z Fa, Y Wan, et al. GLS1 promotes proliferation in hepatocellular carcinoma cells via AKT/GSK3 $\beta /$ CyclinD1 pathway [J]. Experimental Cell Research. 2019; 381:234-43.

61. Mohammad J, RR Singh, C Riggle, B Haugrud, MY Abdalla, et al. JNK inhibition blocks piperlongumine-induced cell death and transcriptional activation of heme oxygenase- 1 in pancreatic cancer cells [J]. Apoptosis. 2019; 24:459-63.

62. Liu L, Y Wu, C Bian, MF Nisar, M Wang, et al. Heme oxygenase 1 facilitates cell proliferation via the B-Raf-ERK signaling pathway in melanoma [J]. Cell Communication and Signaling. 2019; 17:280-95.

63. Nicolás A, S Michael, L Estefania, C Javier, N Nora, et al. Heme Oxygenase-1 Is a Pivotal Modulator of Bone Turnover and Remodeling: Molecular Implications for Prostate Cancer Bone Metastasis [J]. Antioxidants \& redox signaling. 2020; 32:145-62.

64. Kun-Chun C, T Ke-Hung, L Yu-Hsiang, H Chen-Pang, C Kang-Shuo, et al. Antioxidation and Antiapoptosis Characteristics of Heme Oxygenase-1 Enhance Tumorigenesis of Human Prostate Carcinoma Cells [J]. Translational oncology. 2020; 13:677-82.

65. Lv X, DM Song, YH Niu, BS Wang. Inhibition of heme oxygenase-1 enhances the chemosensitivity of laryngeal squamous cell cancer Hep-2 cells to cisplatin [J]. Apoptosis. 2016; 21: 489-501.

66. Sun X, J Li, Y Li, S Wang, Q Li. Apatinib, a Novel Tyrosine Kinase Inhibitor, Promotes ROS-Dependent Apoptosis and Autophagy via the Nrf2/HO-1 Pathway in Ovarian Cancer Cells [J]. Oxid Med Cell Longev. 2020; 2020: 3145182-89.

67. Kim SH, S Saeidi, XC Zhong, SY Gwak, SA Muna, et al. Breast cancer cell debris diminishes therapeutic efficacy through heme oxygenase-1-mediated inactivation of M1-like tumor-associated macrophages [J]. Neoplasia. 2020; 22: 606-16.

68. Kruger C, Y Zhou. Red meat and colon cancer: A review of mechanistic evidence for heme in the context of risk assessment methodology [J]. Food Chem Toxicol. 2018; 118: 131-53.

69. Podkalicka P, O Mucha, S Kruczek, A Biela, K Andrysiak, et al. Synthetically Lethal Interactions of Heme Oxygenase-1 and Fumarate Hydratase Genes [J]. Biomolecules. 2020; 10:781-94.

70. Hui L, Y Chen. Tumor microenvironment: Sanctuary of the devil [J]. Cancer Lett. 2015; 368: 7-13.

71. Wu T, Y Dai. Tumor microenvironment and therapeutic response [J]. Cancer Lett. 2017; 387: 61-68.

72. Leong SP, A Aktipis, C Maley. Cancer initiation and progression within the cancer microenvironment [J]. Clin Exp Metastasis. 2018; 35: 361-67.

73. Hemmati M, B Yousefi, A Bahar, M Eslami. Importance of Heme Oxygenase-1 in Gastrointestinal Cancers: Functions, Inductions, Regulations, and Signaling [J]. J Gastrointest Cancer. 2021;1:340-52.

74. Qiao Q, X Liu, T Yang, K Cui, L Kong, et al. Nanomedicine for acute respiratory distress syndrome: The latest application, targeting strategy, and rational design [J]. Acta Pharm Sin B. 2021;34:119-32.

75. Landis RC, KR Quimby, AR Greenidge. M1/M2 Macrophages in Diabetic Nephropathy: Nrf2/HO-1 as Therapeutic Targets [J]. Curr Pharm Des. 2018; 24: 2241-49.

76. De Wilde V, N Van Rompaey, M Hill, JF Lebrun, P Lemaître, et al. Endotoxin-induced myeloid-derived suppressor cells inhibit alloimmune responses via heme oxygenase-1 [J]. Am J Transplant. 2009; 9: 2034-47.

77. Bogdan C. Macrophages as host, effector and immunoregulatory cells in leishmaniasis: Impact of tissue micro-environment and metabolism [J]. Cytokine X. 2020; 2: 100041-47.

78. Zhang X, S Shi, J Shen, M Zhao, Q He. Functional Immunoregulation by Heme Oxygenase 1 in Juvenile Autoimmune Diseases [J]. Curr Gene Ther. 2019; 19: 110-16.

79. Yang CC, CM Yang. Chinese Herbs and Repurposing Old Drugs as Therapeutic Agents in the Regulation of Oxidative Stress and Inflammation in Pulmonary Diseases [J]. J Inflamm Res. 2021; 14: 657-87.

80. Sun X, Y Liu, C Li, X Wang, R Zhu, et al. Recent Advances of Curcumin in the Prevention and Treatment of Renal Fibrosis [J]. Biomed Res Int. 2017; 2017: 2418671-78.

81. Kumar H, AE Ropper, SH Lee, I Han. Propitious Therapeutic Modulators to Prevent Blood-Spinal Cord Barrier Disruption in Spinal Cord Injury [J]. Mol Neurobiol. 2017; 54: 3578-90.

82. Ayla S, S Karahüseyinogluc. Cancer Stem Cells, Their Microenvironment and Anoikis [J]. Crit Rev Oncog. 2019; 24: 27-34.

83. Jayasooriya RG, SR Park, YH Choi, JW Hyun, WY Chang, et al. Camptothecin suppresses expression of matrix metalloproteinase-9 and vascular endothelial growth factor in DU145 cells through $\mathrm{PI} 3 \mathrm{~K} /$ Akt-mediated inhibition of NF-KB activity and Nrf2-dependent induction of HO-1 expression [J]. Environ Toxicol Pharmacol. 2015; 39: 1189-98.

84. Birrane G, H Li, S Yang, SD Tachado, S Seng. Cigarette smoke induces nuclear translocation of heme oxygenase 1 (HO-1) in prostate cancer cells: nuclear HO-1 promotes vascular endothelial growth factor secretion [J]. Int J Oncol. 2013; 42: 1919-28.

85. Mori N, YS Kida. Expression of genes involved in drug metabolism differs between perfusable 3D liver tissue and conventional 2D-cultured hepatocellular carcinoma cells [J]. FEBS Open Bio. 2020; 10: 1985-2002.

86. Mori N, Y Akagi, Y Imai, Y Takayama, YS Kida. Fabrication of Perfusable Vascular Channels and Capillaries in 3D Liver-like Tissue [J]. Sci Rep. 2020; 10: 5646-58

87. Li Q, Q Liu, WP Cheng, HY Wei, WQ Jiang, et al. Heme Oxygenase-1 Inhibits Tumor Metastasis Mediated by Notch1 Pathway in Murine Mammary Carcinoma [J]. Oncology Research. 2019; 27: 643-51.

88. Victoria MM, S Daiana, GS Martín, C Omar. Regulation of the Expression of Heme Oxygenase-1: Signal Transduction, Gene Promoter Activation, and Beyond [J]. Antioxidants \& redox signaling. 2020; 32:784-94.

89. Khalil HS, SP Langdon, IH Kankia, J Bown, YY Deeni. NRF2 Regulates HER2 and HER3 Signaling Pathway to Modulate Sensitivity to Targeted Immunotherapies [J]. Oxid Med Cell Longev. 2016; 2016: 4148791-97.

90. Wang S, BN Hannafon, J Zhou, WQ Ding. Clofibrate induces heme oxygenase 1 expression through a PPARa-independent mechanism in human cancer cells [J]. Cell Physiol Biochem. 2013; 32: 1255-64.

91. Su J, F Zhang, X Li, Z Liu. Osthole promotes the suppressive effects of cisplatin on NRF2 expression to prevent drug-resistant cervical cancer progression [J]. Biochem Biophys Res Commun. 2019; 514: 510-17.

92. Sahin K, E Yenice, M Tuzcu, C Orhan, C Mizrak, et al. Lycopene Protects Against Spontaneous Ovarian Cancer Formation in Laying Hens [J]. J Cancer Prev. 2018; 23: 25-36.

93. Sahin K, C Orhan, M Tuzcu, N Sahin, H Tastan, et al. Chemopreventive and Antitumor Efficacy of Curcumin in a Spontaneously Developing Hen Ovarian Cancer Model [J]. Cancer Prev Res (Phila). 2018; 11: 59-67.

94. Sun TC, XC Liu, SH Yang, LL Song, SJ Zhou, et al. Melatonin Inhibits Oxidative Stress and Apoptosis in Cryopreserved Ovarian Tissues via Nrf2/ HO-1 Signaling Pathway [J]. Front Mol Biosci. 2020; 7: 163-78.

95. Yang J, X Zhao, M Tang, L Li, Y Lei, et al. The role of ROS and subsequent DNA-damage response in PUMA-induced apoptosis of ovarian cancer cells [J]. Oncotarget. 2017; 8: 23492-506.

96. Sun X, S Wang, J Gai, J Guan, J Li, et al. SIRT5 Promotes Cisplatin Resistance in Ovarian Cancer by Suppressing DNA Damage in a ROS-Dependent Manner via Regulation of the Nrf2/HO-1 Pathway [J]. Front Oncol. 2019; 9: 754-65.

97. Yong M, T Yu, S Tian, S Liu, J Xu, et al. DR2 blocker thioridazine: A promising drug for ovarian cancer therapy [J]. Oncol Lett. 2017; 14: 8171-77.

98. Yang S, D Zhang, N Shen, G Wang, Z Tang, et al. Dihydroartemisinin increases gemcitabine therapeutic efficacy in ovarian cancer by inducing reactive oxygen species [J]. J Cell Biochem. 2019; 120: 634-44.

99. Iwabuchi T, C Yoshimoto, H Shigetomi, H Kobayashi. Oxidative Stress and Antioxidant Defense in Endometriosis and Its Malignant Transformation [J]. Oxid Med Cell Longev. 2015; 2015: 848595-98.

100. Yamada Y, T Uchiyama, F Ito, N Kawahara, K Ogawa, et al. Clinical significance of M2 macrophages expressing heme oxygenase-1 in malignant transformation of ovarian endometrioma [J]. Pathol Res Pract. 2019; 215: 639-43

101. Yang Y, HX Wang, L Zhang, W Huo, XD Li, et al. Inhibition of Heme Oxygenase-1 enhances hyperthermia-induced autophagy and antiviral effect [J]. Int J Biol Sci. 2019; 15: 568-78.

102. Travaglino A, A Raffone, G Saccone, A Mollo, G De Placido, et al. Endometrial hyperplasia and the risk of coexistent cancer: WHO versus EIN criteria [J]. Histopathology. 2019; 74: 676-87.

103. Mills AM, TA Longacre. Endometrial hyperplasia [J]. Semin Diagn Pathol. 2010; 27: 199-214.

104. Sanderson PA, HO Critchley, AR Williams, MJ Arends, PT Saunders. New concepts for an old problem: the diagnosis of endometrial hyperplasia [J]. Hum Reprod Update. 2017; 23: 232-54.

105. Xue J, S Wang, J Wu, BN Hannafon, WQ Ding. Zinc at sub-cytotoxic concentrations induces heme oxygenase- 1 expression in human cancer cells [J]. Cell Physiol Biochem. 2013; 32: 100-107.

106. Decollogne S, S Joshi, SA Chung, PP Luk, RX Yeo, et al. Alterations in the mitochondrial responses to PENAO as a mechanism of resistance in ovarian cancer cells [J]. Gynecol Oncol. 2015; 138: 363-71.

107. Jiao Y, BN Hannafon, RR Zhang, KM Fung, WQ Ding. Docosahexaenoic acid and disulfiram act in concert to kill cancer cells: a mutual enhancement of their anticancer actions [J]. Oncotarget. 2017; 8: 17908-20. 
108. Fu-Tai S, H Liang-Liang, A Xu-Sheng, Z Xiang-Cheng, G Shi-Guang, et al. ZnPPIX inhibits peritoneal metastasis of gastric cancer via its antiangiogenic activity [J]. Biomedicine \& pharmacotherapy = Biomedecine \& pharmacotherapie. 2015; 71:465-73

109. Wang S, JE Avery, BN Hannafon, SE Lind, WQ Ding. Zinc protoporphyrin suppresses cancer cell viability through a heme oxygenase-1-independent mechanism: the involvement of the Wnt/ $\beta$-catenin signaling pathway [J]. Biochem Pharmacol. 2013; 85: 1611-18.

110. Shuai W, HB N, LS E, D Wei-Qun. Zinc Protoporphyrin Suppresses $\beta$-Catenin Protein Expression in Human Cancer Cells: The Potential Involvement of Lysosome-Mediated Degradation [J]. PloS one. 2015; 10:176-83

111. Yong SB, J Kim, JY Chung, S Ra, SS Kim, et al. Heme Oxygenase 1Targeted Hybrid Nanoparticle for Chemo- and Immuno-Combination Therapy in Acute Myelogenous Leukemia [J]. Advanced Science. 2020; 7:121-32

112. Floresta G, V Pittalà, V Sorrenti, G Romeo, L Salerno, et al. Development of new HO-1 inhibitors by a thorough scaffold-hopping analysis [J]. Bioorg Chem. 2018; 81: 334-39.

113. Kato I, T Kasukabe, S Kumakura. Menin-MLL inhibitors induce ferroptosis and enhance the anti-proliferative activity of auranofin in several types of cancer cells [J]. Int J Oncol. 2020; 57: 1057-71. 